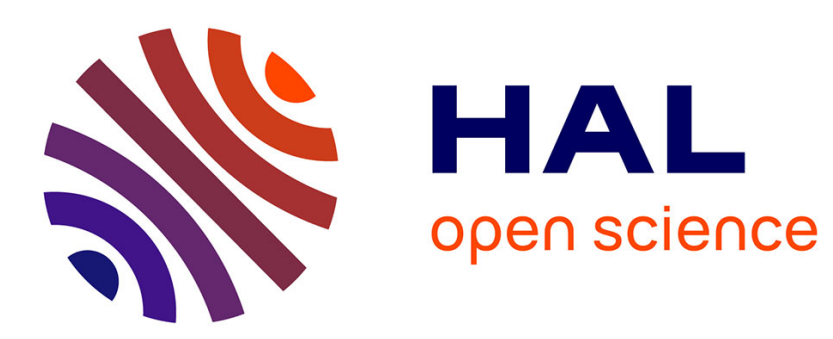

\title{
A Cut-Free Simple Sequent Calculus for Modal Logic S5 Francesca Poggiolesi
}

\section{To cite this version:}

Francesca Poggiolesi. A Cut-Free Simple Sequent Calculus for Modal Logic S5. The review of symbolic logic, 2008, 1 (1), pp.3-15. halshs-00775809

\section{HAL Id: halshs-00775809 \\ https://shs.hal.science/halshs-00775809}

Submitted on 26 Jun 2018

HAL is a multi-disciplinary open access archive for the deposit and dissemination of scientific research documents, whether they are published or not. The documents may come from teaching and research institutions in France or abroad, or from public or private research centers.
L'archive ouverte pluridisciplinaire HAL, est destinée au dépôt et à la diffusion de documents scientifiques de niveau recherche, publiés ou non, émanant des établissements d'enseignement et de recherche français ou étrangers, des laboratoires publics ou privés. 


\title{
Francesca Poggiolesi
}

\section{A Cut-Free Simple Sequent Calculus for Modal Logic S5}

\begin{abstract}
In this paper we present a simple sequent calculus for the modal propositional logic S5. We prove that this sequent calculus is theoremwise equivalent to the Hilbert-style system S5, that it is contraction-free and cut-free, and finally that it is decidable. All results are proved in a purely syntactic way.
\end{abstract}

\section{Introduction}

Amongst the many normal systems of modal propositional logic, one of the most important and well-known is doubtlessly S5.

When considered from the point of view of Kripke semantics, S5 is quite a peculiar system since it can be described in two different but equivalent ways ${ }^{1}$. The first one specifies the properties that the accessibility relation between worlds of a Kripke frame should satisfy: S5 is indeed sound and complete with respect to the class of reflexive, transitive and symmetric frames (or, equivalently, in the class of reflexive and euclidean frames). A second and easier way to study S5 semantically exploits Kripke frames where the accessibility relation is absent ${ }^{2}$ : S5 is indeed sound and complete with respect to the class of frames which are just non-empty sets of worlds.

This second way is evidently simpler and it would be useful and interesting to reflect this simplicity at the syntactic level, within, for example, a Gentzen system. Unfortunately this has not yet been achieved: indeed, it turns out to be quite a challenge to give a sequent calculus for S5. The efforts in this direction are numerous and each of them presents some difficulties. The most common fault of the sequent calculi proposed so far is not being cut-free (Blumey and Humberstone (1991), Matsumoto and Ohnishi (1959)), or, in any case, not satisfying the subformula property (Negri (2005), Sato (1980), and Wansing (1994)). Other Gentzen calculi are syntactically impure because they use explicit semantic parameters (Braüner (2000), Cerrato (1993), Mints (1997)). Finally there exist Gentzen systems for S5 which are quite laborious since they treat S5 as a system whose accessibility relation satisfies several conditions (Brünnler (2006), Došen (1985), Indrezejczak (1997)).

The best solutions are probably those offered by Avron (1996) and Restall (2006): both these solutions (i) use hypersequents, (ii) try to reflect the simpler

\footnotetext{
${ }^{1}$ For a detailed explanation of this point see Restall (2006).

${ }^{2}$ From now on, we will call this kind of frame an S5 Kripke frame.
} 
way S5 can be described semantically. Since the main goal of this paper is to present a new sequent calculus for S5 which shares with Avron and Restall's solutions the points (i) and (ii), it is worth explaining in detail how our approach differs from these two. The major differences are the following. Firstly, in our calculus all the structural rules are (height-preserving) admissible (even the contraction-rules), and the logical and modal rules are height-preserving invertible. Secondly, the rules which compose our calculus are different from the rules of the other calculi. Thirdly, we prove the decidability theorem in a purely syntactic way. Finally, as has been shown in Poggiolesi (2007), our calculus is closely related to the so called tree-hypersequents method (Poggiolesi (2008)) by means of which we can obtain Gentzen systems for several modal logics. Like Restall, but in contrast to Avron, our rules are explicit ${ }^{3}$. Finally we point out that Restall is the only one to propose a proofnets interpretation of his sequent calculus.

\section{The calculus $\mathrm{CSS5}_{s}$}

We define the modal propositional language $\mathcal{L}^{\square}$ in the following way:

- atoms: $p_{0}, p_{1}, \ldots$

- logical constant: $\square$,

- connectives: $\neg, \vee$.

The other connectives, the modal operator $\diamond$, and the formulas of the modal language $\mathcal{L}^{\square}$ are defined as usual.

Syntactic Conventions:

$-\alpha, \beta, \ldots:$ formulas,

- $M, N, P, Q, \ldots$ : finite multisets of formulas,

$-\Gamma, \Delta, \ldots$ : classical sequents.

- $G, H, \ldots$ : hypersequents.

As we will deal with hypersequents, we remind the reader what a hypersequent is.

Definition 2.1. A hypersequent is a syntactic object of the form:

$$
M_{1} \Rightarrow N_{1}\left|M_{2} \Rightarrow N_{2}\right| \ldots \mid M_{n} \Rightarrow N_{n}
$$

where $M_{i} \Rightarrow N_{i}(i=1, \ldots, n)$ are classical sequents.

\footnotetext{
${ }^{3}$ Intuitively, the rules of a sequent calculus are explicit when they can be presented in such a way that the symbol they introduce does not appear in their premise(s). For a definition of the explicitness property see Wansing (1994).
} 
Definition 2.2. The intended interpretation of a hypersequent is defined inductively in the following way:

$$
\begin{aligned}
& \text { - }(M \Rightarrow N)^{\tau}:=\bigwedge M \rightarrow \bigvee N, \\
& \text { - }\left(\Gamma_{1}\left|\Gamma_{2}\right| \ldots \mid \Gamma_{n}\right)^{\tau}:=\square \Gamma_{1}^{\tau} \vee \square \Gamma_{2}^{\tau} \vee \ldots \vee \square \Gamma_{n}^{\tau}
\end{aligned}
$$

Given the definition and the interpretation of the notion of hypersequent, it should be clear that a hypersequent is just a multiset of classical sequents, which is to say that the order of the sequents in a hypersequents is not important.

The postulates of the calculus $C S S 5_{s}{ }^{4}$ are:

\section{Initials Hypersequents}

$G \mid M, p \Rightarrow N, p$

\section{Propositional Rules}

$$
\begin{array}{lc}
\frac{G \mid M \Rightarrow N, \alpha}{G \mid \neg \alpha, M \Rightarrow N} \neg A & \frac{G \mid \alpha, M \Rightarrow N}{G \mid M \Rightarrow N, \neg \alpha} \neg K \\
\frac{G \mid \alpha, \beta, M \Rightarrow N}{G \mid \alpha \wedge \beta, M \Rightarrow N} \wedge A & \frac{G|M \Rightarrow N, \alpha \quad G| M \Rightarrow N, \beta}{G \mid M \Rightarrow N, \alpha \wedge \beta} \wedge K
\end{array}
$$

\section{Modal Rules}

$$
\begin{array}{ll}
\frac{G \mid \alpha, \square \alpha, M \Rightarrow N}{G \mid \square \alpha, M \Rightarrow N} \square A_{1} & G|M \Rightarrow N| \Rightarrow \alpha \\
\frac{G|\square \alpha, M \Rightarrow N| \alpha, P \Rightarrow Q}{G|\square \alpha, M \Rightarrow N| P \Rightarrow Q} \square A_{2} &
\end{array}
$$

Let us make two remarks on the modal rules. The first one only concerns the rules $\left(\square A_{i}\right)(i=1,2)$. The repetition of the principal formula $\square \alpha$ in the premise of each of these rules only serves to make the rules invertible. This is analogous to the repetition of the formula $(\forall x)(\alpha(x))$ in the premise of the rule which

\footnotetext{
${ }^{4}$ The name $C S S 5_{s}$ stands for: simple version of the calculus CSS5. Indeed in Poggiolesi (2006) we have introduced another sequent calculus for $S 5$, called precisely $C S S 5$, which reflects the more complicated way S5 can be described semantically that was mentioned in the introduction.
} 
introduces the symbol $\forall$ on the left side of the sequent in some versions of the sequent calculus for first-order classical logic.

The second remark concerns the three modal rules. It is easy to informally understand these rules if we compare the hypersequent to a S5 Kripke frame, and the sequents which compose the hypersequent to different worlds of the S5 Kripke frame. In this perspective, the rule $(\square K)$ says, if read bottom up, that, if the formula $\square \alpha$ is false at a world $x$, then we can create a new world $y$ where the formula $\alpha$ is false; on the other hand, the rules $\left(\square A_{i}\right)$ tell us, if read bottom up and considered together, that, if the formula $\square \alpha$ is true at a world $x$, then the formula $\alpha$ is true in any world of the frame.

\section{Admissibility of the Structural Rules}

In this section we will show which structural rules are admissible in the calculus $C S S 5_{s}$. Moreover, in order to show that the two rules of contraction are heightpreserving admissible, we will show that all the logical and modal rules are height-preserving invertible. The proof of the admissibility of the cut-rule will be shown in the fifth section.

Definition 3.1. We associate to each proof $d$ in $C S S 5_{s}$ a natural number $h(d)$ (the height). Intuitively, the height corresponds to the length of the longest branch in a tree-proof $d$, minus one. We define $h(d)$ by induction on the construction of $d$.

$$
\begin{aligned}
& d \equiv \quad G \mid M \Rightarrow N: \quad h(d)=0 \\
& d \equiv \frac{\left.G^{\prime} \mid M^{\prime} \Rightarrow N^{\prime}\right\}^{d_{1}}}{G \mid M \Rightarrow N} \mathcal{R}: \\
& h(d)=h\left(d_{1}\right)+1 \\
& d \equiv \frac{{ }^{d_{1}}\left\{G\left|M^{\prime} \Rightarrow N^{\prime} \quad G\right| M^{\prime \prime} \Rightarrow N^{\prime \prime}\right\}^{d_{2}}}{G \mid M \Rightarrow N}{ }_{\mathcal{R}}: \quad h(d)=\max \left(h\left(d_{1}\right)+1, h\left(d_{2}\right)+1\right)
\end{aligned}
$$

Definition 3.2. $d \vdash^{n} G$ means that $d$ is a proof of $G$ in $C S S 5_{s}$, with $h(d) \leq n$. We write $\vdash^{\langle n\rangle} G$, or just ${ }^{\langle n\rangle} G$, for: "there exists a proof $d$ such that $d \vdash^{n} G$."

Definition 3.3. Let $G$ be a hypersequent and $G^{\prime}$ be the result of the application of a certain rule $\mathcal{R}$ on $G$. We say that this rule $\mathcal{R}$ is height-preserving admissible when: 


$$
d \vdash^{n} G \quad \Rightarrow \quad \exists d^{\prime}\left(d^{\prime} \vdash^{n} G^{\prime}\right)
$$

We call a rule, $\mathcal{R}$, which transforms a hypersequent $G$ into a hypersequent $G^{\prime}$, admissible when:

$$
d \vdash^{n} G \quad \Rightarrow \quad \exists d^{\prime}\left(d^{\prime} \vdash G^{\prime}\right)
$$

Lemma 3.4. Hypersequents of the form $G \mid \alpha, M \Rightarrow N, \alpha$, with $\alpha$ an arbitrary modal formula, are derivable in $C S S 5_{s}$.

Proof. By straightforward induction on $\alpha . \quad \otimes$

Lemma 3.5. The rule of merge:

$$
\frac{G|M \Rightarrow N| P \Rightarrow Q}{G \mid M, P \Rightarrow N, Q}{ }_{\text {merge }}
$$

is height-preserving admissible in $C S S 5_{s}$.

Proof. By induction on the height of the derivation of the premise.

If the premise is an initial hypersequent, then so is the conclusion.

If the premise is inferred by a logical rule, then the inference is clearly preserved. We will give an example using the logical rule $(\neg K)$ :

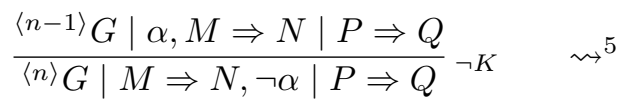

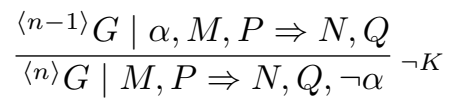

If the premise is inferred by the modal rule ( $\square K)$, this is clearly preserved:

$$
\begin{aligned}
& \frac{\left\langle{ }^{\langle n-1\rangle} G|M \Rightarrow N| P \Rightarrow Q\right| \Rightarrow \alpha}{{ }^{\langle n\rangle} G|M \Rightarrow N, \square \alpha| P \Rightarrow Q} \square K \\
& \frac{\langle n-1\rangle G|M, P \Rightarrow N, Q| \Rightarrow \alpha}{{ }^{\langle n\rangle} G \mid M, P \Rightarrow N, Q, \square \alpha} \square K
\end{aligned}
$$

${ }^{5}$ The symbol $\rightsquigarrow$ means: the premise of the right side is obtained by induction hypothesis on the premise of the left side. 
If the premise is inferred by the modal rule $\left(\square A_{1}\right)$, this is clearly preserved:

$$
\begin{aligned}
& \frac{\left\langle{ }^{(n-1\rangle} G|\alpha, \square \alpha, M \Rightarrow N| P \Rightarrow Q\right.}{{ }^{\langle n\rangle} G|\square \alpha, M \Rightarrow N| P \Rightarrow Q} \square A_{1} \\
& \frac{{ }^{\langle n-1\rangle} G \mid \square \alpha, \alpha, M, P \Rightarrow N, Q}{{ }^{\langle n\rangle} G \mid \square \alpha, M, P \Rightarrow N, Q} \square A_{1}
\end{aligned}
$$

If the premise is inferred by the modal rule $\left(\square A_{2}\right)$, there are two significant cases to analyze: the one where the rule $\left(\square A_{2}\right)$ has been applied between the two sequents $M \Rightarrow N$ and $P \Rightarrow Q$; the one where the rule $\left(\square A_{2}\right)$ has been applied between a third sequent, let us call it $\Gamma$, and $M \Rightarrow N$ (or, equivalently, $P \Rightarrow Q$ ). The two situations are analogous, therefore we only analyze the first one in detail:

$$
\begin{aligned}
& \frac{\langle n-1\rangle}{{ }^{\langle n}|\square \alpha, M \Rightarrow N| \alpha, P \Rightarrow Q} \square A_{2} \\
& \frac{{ }^{\langle n-1\rangle} G \mid \square \alpha, \alpha, M, P \Rightarrow N, Q}{{ }^{\langle n\rangle} G \mid \square \alpha, M, P \Rightarrow N, Q} \square A_{1}
\end{aligned}
$$

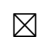

Lemma 3.6. The rule of external weakening:

$$
\frac{G}{G \mid M \Rightarrow N} E W
$$

is height-preserving admissible in $C S S 5_{s}$.

Proof. By straightforward induction on the height of the derivation of the premise. $\otimes$

Lemma 3.7. The rule of internal weakening:

$$
\frac{G \mid M \Rightarrow N}{G \mid M, P \Rightarrow N, Q} I W
$$

is height-preserving admissible in $C S S 5_{s}$. 
Proof. It follows by the height-preserving admissibility of the two rules of merge and external weakening.

$\bigotimes$

Lemma 3.8. All the logical and modal rules of $C S S 5_{s}$ are height-preserving invertible.

Proof. The proof proceeds by induction on the height of the derivation of the premise of the rule considered. The cases of logical rules are dealt with in the classical way. The only differences - the fact that we are dealing with hypersequents and the cases where the last applied rule is one of the rules $\left(\square A_{i}\right)$ or $(\square K)$ - are dealt with easily.

The rules $\left(\square A_{i}\right)$ are trivially height-preserving invertible since both their premises are obtained by weakening from their respective conclusions, and weakening is height-preserving admissible.

We show in detail the invertibility of the rule ( $\square K)$. If $G \mid M \Rightarrow N, \square \alpha$ is an initial hypersequent, then so is $G|M \Rightarrow N| \Rightarrow \alpha$. If $G \mid M \Rightarrow N, \square \alpha$ is obtained by a logical rule $\mathcal{R}$, we apply the inductive hypothesis on the premise(s), $G^{\prime} \mid M^{\prime} \Rightarrow N^{\prime}, \square \alpha\left(G^{\prime \prime} \mid M^{\prime \prime} \Rightarrow N^{\prime \prime}, \square \alpha\right)$ and we obtain derivation(s), of height $n-1$, of $G^{\prime}\left|M^{\prime} \Rightarrow N^{\prime}\right| \Rightarrow \alpha\left(G^{\prime \prime}\left|M^{\prime \prime} \Rightarrow N^{\prime \prime}\right| \Rightarrow \alpha\right)$. By applying the rule $\mathcal{R}$, we obtain a derivation of height $n$ of $G|M \Rightarrow N| \Rightarrow \alpha$. If $G \mid M \Rightarrow N, \square \alpha$ is of the form $G \mid \square \beta, M^{\prime} \Rightarrow N, \square \alpha$, then it may have been obtained by the two modal rules $\left(\square A_{i}\right)$. Since the procedure is the same in both cases, we can just analyze the one of the rule $\left(\square A_{1}\right)$ and the other can be dealt with analogously. We apply the inductive hypothesis on $G \mid \square \beta, \beta, M^{\prime} \Rightarrow N, \square \alpha$ and we obtain a derivation of height $n-1$ of $G\left|\square \beta, \beta, M^{\prime} \Rightarrow N\right| \Rightarrow \alpha$. By applying the rule $\left(\square A_{1}\right)$, we obtain a derivation of height $n$ of $G\left|\square \beta, M^{\prime} \Rightarrow N\right| \Rightarrow \alpha$. If $G \mid M \Rightarrow N, \square \alpha$ is obtained by the modal rule $(\square K)$ in which $\square \alpha$ is not the principal formula, then this case can be treated analogously to the one of $\left(\square A_{i}\right)$. Finally, if $G \mid M \Rightarrow N, \square \alpha$ is obtained by the modal rule ( $\square K)$ and $\square \alpha$ is the principal formula, the premise of the last step gives the conclusion. $\square$

Lemma 3.9. The rules of contraction:

$$
\frac{G \mid \alpha, \alpha, M \Rightarrow N}{G \mid \alpha, M \Rightarrow N} C A \quad \frac{G \mid M \Rightarrow N, \alpha, \alpha}{G \mid M \Rightarrow N, \alpha} C K
$$

are height-preserving admissible in $C S S 5_{s}$.

Proof. By induction on the height of the derivation of the premise $G \mid M \Rightarrow$ $N, \alpha, \alpha(G \mid \alpha, \alpha, M \Rightarrow N)$. We analyze only the case of the rule $C K$. The case of the rule $C A$ is symmetric.

If $G \mid M \Rightarrow N, \alpha, \alpha$ is an initial hypersequent, so is $G \mid M \Rightarrow N, \alpha$.

If $G \mid M \Rightarrow N, \alpha, \alpha$ is the conclusion of a rule $\mathcal{R}$ which does not have either of the two occurrences of the formula $\alpha$ as principal, we apply the inductive 
hypothesis on the premise(s) $G^{\prime} \mid M^{\prime} \Rightarrow N^{\prime}, \alpha, \alpha\left(G^{\prime \prime} \mid M^{\prime \prime} \Rightarrow N^{\prime \prime}, \alpha, \alpha\right)$, obtaining derivation(s) of height $n-1$ of $G^{\prime} \mid M^{\prime} \Rightarrow N^{\prime}, \alpha\left(G^{\prime \prime} \mid M^{\prime \prime} \Rightarrow N^{\prime \prime}, \alpha\right)$. By applying the rule $\mathcal{R}$ we obtain a derivation of height $n$ of $G \mid M \Rightarrow N, \alpha$.

$G \mid M \Rightarrow N, \alpha, \alpha$ is the conclusion of a logical or modal rule and one of the two occurrences of the formula $\alpha$ is principal. Hence the last rule used in the proof of $G \mid M \Rightarrow N, \alpha, \alpha$ is a $K$-rule and we have to analyze the following three cases: $\neg K, \wedge K, \square K$.

$[\neg K]:$

$$
\frac{{ }^{\langle n-1\rangle} G \mid \beta, M \Rightarrow N, \neg \beta}{{ }^{\langle n\rangle} G \mid M \Rightarrow N, \neg \beta, \neg \beta} \neg K \quad \rightarrow{ }^{6} \quad \frac{{ }^{\langle n-1\rangle} G \mid \beta, \beta, M \Rightarrow N}{{ }^{\langle n-1\rangle} G \mid \beta, M \Rightarrow N} i . h .
$$

$[\wedge K]:$

$$
\begin{aligned}
& \frac{\langle n-1\rangle G|M \Rightarrow N, \beta, \beta \wedge \gamma \quad\langle n-1\rangle G| M \Rightarrow N, \gamma, \beta \wedge \gamma}{{ }^{\langle n\rangle} G \mid M \Rightarrow N, \beta \wedge \gamma, \beta \wedge \gamma} \wedge K \rightarrow
\end{aligned}
$$

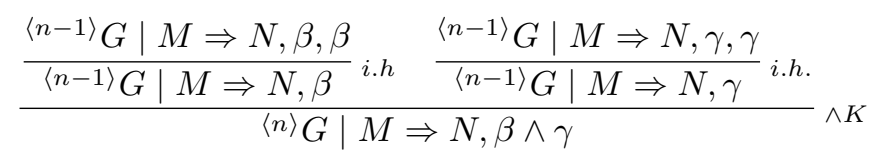

$[\square K]:$

$$
\begin{aligned}
& \frac{\langle n-1\rangle}{{ }^{\langle n} G|M \Rightarrow N, \square \beta| \Rightarrow \beta} \square \quad-\rightarrow \\
& \frac{{ }^{\langle n-1\rangle} G|M \Rightarrow N| \Rightarrow \beta \mid \Rightarrow \beta}{\frac{}{\langle n-1\rangle} G|M \Rightarrow N| \Rightarrow \beta, \beta_{\text {merge }}^{\langle n-1\rangle} G|M \Rightarrow N| \Rightarrow \beta} \text { me.h. }
\end{aligned}
$$

$\nabla$

\footnotetext{
${ }^{6}$ The symbol $\rightarrow$ means: the premise of the right side is obtained by an application of Lemma 3.8 on the premise of the left side.
} 


\section{The Adequateness Theorem}

In this section we briefly prove that the sequent calculus $C S S 5_{s}$ proves exactly the same formulas as its corresponding Hilbert-style system $S 5$.

\section{Theorem 4.1.}

(i) If $\vdash \alpha$ in $S 5$, then $\vdash \Rightarrow \alpha$ in $C S S 5_{s}$.

(ii) If $\vdash G$ in $C S S 5_{s}$, then $\vdash(G)^{\tau}$ in $S 5$.

Proof. By induction on the height of proofs in $S 5$ and $C S S 5_{s}$, respectively. In order to further acquaint the reader with the calculus $C S S 5_{s}$ we verify (i). The classical axioms and the modus ponens are proved as usual, we just present the proof of the axiom $T$, the axiom 4 , the axiom $B$ and the axiom 5 .

$C S S 5_{s} \vdash \Rightarrow \square \rightarrow \alpha$

$$
\frac{\square \alpha, \alpha \Rightarrow \alpha}{\square \alpha \Rightarrow \alpha}
$$

$C S S 5_{s} \vdash \Rightarrow \square \rightarrow \square \square \alpha$

$$
\begin{array}{r}
\square \alpha \Rightarrow|\Rightarrow| \alpha \Rightarrow \alpha \\
\frac{\square \alpha \Rightarrow|\Rightarrow| \Rightarrow \alpha}{\square \alpha \Rightarrow \mid \Rightarrow \square \alpha} \\
\frac{\square \alpha \Rightarrow \square \square \alpha}{\square \alpha} \\
\Rightarrow \square \alpha \rightarrow \square \square \alpha
\end{array}
$$

$C S S 5_{s} \vdash \alpha \rightarrow \square \neg \square \neg \alpha$

$$
\begin{gathered}
\frac{\alpha \Rightarrow \alpha \mid \square \neg \alpha \Rightarrow}{\neg \alpha, \alpha \Rightarrow \mid \square \neg \alpha \Rightarrow} \\
\frac{\alpha \Rightarrow \mid \square \neg \alpha \Rightarrow}{\alpha \Rightarrow \mid \Rightarrow \neg \square \neg \alpha} \\
\frac{\alpha \Rightarrow \square \neg \neg \alpha}{\alpha \Rightarrow \square \rightarrow \square \square}
\end{gathered}
$$

$C S S 5_{s} \vdash \Rightarrow \square \neg \alpha \rightarrow \square \neg \square \neg \alpha$ 


$$
\begin{gathered}
\Rightarrow|\square \neg \alpha \Rightarrow| \alpha \Rightarrow \alpha \\
\Rightarrow|\square \neg \alpha \Rightarrow| \Rightarrow \neg \alpha, \alpha \\
\Rightarrow|\square \neg \alpha \Rightarrow| \neg \alpha \Rightarrow \neg \alpha \\
\hline \Rightarrow|\square \neg \alpha \Rightarrow| \Rightarrow \neg \alpha \\
\frac{\Rightarrow|\Rightarrow \neg \square \neg \alpha| \Rightarrow \neg \alpha}{\Rightarrow \square \neg \alpha \mid \Rightarrow \neg \square \neg \alpha} \\
\frac{\Rightarrow \square \neg \alpha, \square \neg \square \neg \alpha}{\Rightarrow \square \neg \alpha \Rightarrow \square \neg \square \neg \alpha} \\
\Rightarrow \neg \square \neg \alpha \rightarrow \square \neg \square \neg \alpha
\end{gathered}
$$

\section{Cut-elimination Theorem}

In this section we prove the cut-elimination theorem for the calculus $C S S 5_{s}$.

Theorem 5.1. Let $G \mid M \Rightarrow N, \alpha$ and $G^{\prime} \mid \alpha, P \Rightarrow Q$ be two hypersequents, we want to prove that:

$$
\text { if } \frac{\begin{array}{c}
\vdots \\
d_{1}\left\{G\left|M \Rightarrow N, \alpha \quad G^{\prime}\right| \alpha, P \Rightarrow Q\right\}^{d_{2}}
\end{array}}{\text { cut }_{\alpha}}
$$

and $d_{1}$ and $d_{2}$ do not contain any other application of the cut-rule, then we can construct a proof of $G\left|G^{\prime}\right| M, P \Rightarrow N, Q$ without any application of the cut-rule.

Proof. This is proved by induction on the complexity of the cut-formula, which is the number $(\geq 0)$ of the occurrences of logical symbols in cut formula $\alpha$, with subinduction on the sum of the heights of the derivations of the premises of the cut. We will distinguish cases by the last rule applied on the left premise.

Case 1. $G \mid M \Rightarrow N, \alpha$ is an initial hypersequent. Then either the conclusion is also an initial hypersequent or the cut can be replaced by various applications of the internal and external weakening rules on $G^{\prime} \mid \alpha, P \Rightarrow Q$.

Case 2. $G \mid M \Rightarrow N, \alpha$ is inferred by a rule $\mathcal{R}$ in which $\alpha$ is not principal. The reduction is carried out in the standard way by induction on the sum of the heights of the derivations of the premises of cut.

Case 3. $G \mid M \Rightarrow N, \alpha$ is inferred by a rule $\mathcal{R}$ in which $\alpha$ is principal. We distinguish two subcases: in one subcase $\mathcal{R}$ is a propositional rule, in the other $\mathcal{R}$ is a modal rule. 
Case 3.1. As an example, we consider the case where the rule before $G \mid M \Rightarrow$ $N, \alpha$ is $\neg K$, we have:

$$
\frac{\frac{G \mid \beta, M \Rightarrow N}{G \mid M \Rightarrow N, \neg \beta} \neg \quad G^{\prime} \mid \neg \beta, P \Rightarrow Q}{\text { cut }_{\neg \beta}}
$$

By applying lemma 3.8 on $G^{\prime} \mid \neg \beta, P \Rightarrow Q$, we obtain $G^{\prime} \mid P \Rightarrow Q, \beta$. Therefore we can replace the previous cut with the following one which is eliminable by induction on the complexity of the cut formula:

$$
\frac{G^{\prime}|P \Rightarrow Q, \beta \quad G| \beta, M \Rightarrow N}{G\left|G^{\prime}\right| M, P \Rightarrow N, Q} \text { cut }_{\beta}
$$

Case 3.2. $\mathcal{R}$ is $\square K$ and $\alpha \equiv \square \beta$. We have the following situation:

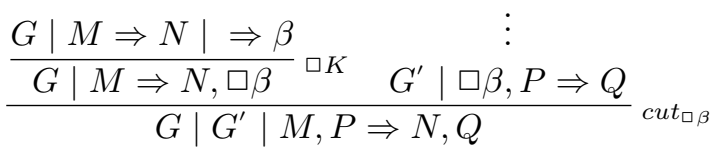

We have to consider the last rule $\mathcal{R}^{\prime}$ of $d_{2}$. If there is no rule $\mathcal{R}^{\prime}$ which introduces $G^{\prime} \mid \square \beta, P \Rightarrow Q$ because $G^{\prime} \mid \square \beta, P \Rightarrow Q$ is an initial hypersequent, then we can solve the case as in 1 . If $\mathcal{R}^{\prime}$ is a rule in which $\square \beta$ is not principal, we solve the case as in 2 . The only problematic cases are thus those in which $\mathcal{R}^{\prime}$ is one of the rules $\left(\square A_{i}\right)$. Since the procedure is the same in both cases, we need only analyze the case of the rule $\left(\square A_{2}\right)$; the other case is dealt with analogously.

$$
\frac{\frac{G|M \Rightarrow N| \Rightarrow \beta}{G \mid M \Rightarrow N, \square \beta} \square K \quad \frac{G^{\prime}|\square \beta, P \Rightarrow Q| \beta, Z \Rightarrow W}{G^{\prime}|\square \beta, P \Rightarrow Q| Z \Rightarrow W} \square A_{2}}{G\left|G^{\prime}\right| M, P \Rightarrow N, Q \mid Z \Rightarrow W} \text { cut } \square \beta
$$

We reduce to:

$$
\begin{aligned}
& \frac{G\left|M \Rightarrow N, \square \beta \quad G^{\prime}\right| \square \beta, P \Rightarrow Q \mid \beta, Z \Rightarrow W}{G\left|G^{\prime}\right| M, P \Rightarrow N, Q \mid \beta, Z \Rightarrow W}{c u t_{\square \beta}} \\
& \frac{G|M \Rightarrow N| \Rightarrow \beta \quad G\left|G^{\prime}\right| M, P \Rightarrow N, Q \mid \beta, Z \Rightarrow W}{\text { cut }_{\beta}}
\end{aligned}
$$


where the first cut is eliminable by induction on the sum of the heights of the derivations of the premises of cut and the second cut is eliminable by induction on the complexity of cut formula. $\otimes$

\section{Decidability}

In this section we prove that the calculus $C S S 5_{s}$ is decidable, which is to say there is an algorithm that, given any hypersequent $G$, determines whether $G$ is provable in $C S S 5_{s}$ or not.

First of all let us observe that our calculus satisfies the subformula property since: (i) the cut rule is admissible in it (see theorem 5.1), (ii) in each of its rules all the formulas which occur in the premise(s) are subformulas of the formulas which occur in the conclusion. Moreover it can also be shown that the contraction rules are admissible (see lemma 3.9). It would therefore seem that any source of potentially non-terminating proof search had been cut off. Unfortunately it is not so because of the repetition of the principal formula in each of the rules $\left(\square A_{i}\right)$. In order to avoid this problem and prove that our calculus is decidable, we shall obtain a bound on the number of applications of the rules $\left(\square A_{i}\right)$.

For this goal, let us start by only taking into account minimal derivations, which is to say, derivations where shortenings are not possible. Then we prove, by means of the following lemmas and their corollaries, that in minimal derivations it is enough to apply the rule $\left(\square A_{1}\right)$ only once on any given pair of principal formulas and the rule $\left(\square A_{2}\right)$ only once on any given pair of sequents. This technique is inspired by the one used in Negri (2005).

Lemma 6.1. The rule $\left(\square A_{1}\right)$ permutes down with respect to the rules $(\neg A)$, $(\neg K),(\wedge A),(\wedge K),\left(\square A_{2}\right)$ and $(\square K)$.

Proof. Let us first of all consider the permutation with one-premise logical rules, which is straightforward. Taking, as an example, the case of the rule $(\neg K)$, we have:

$$
\begin{aligned}
& \frac{G \mid \beta, \alpha, \square \alpha, M \Rightarrow N}{\frac{G \mid \beta, \square \alpha, M \Rightarrow N}{G \mid \square \alpha, M \Rightarrow N, \neg \beta} \neg K} \square A_{1} \\
& \downarrow \\
& \frac{G \mid \beta, \alpha, \square \alpha, M \Rightarrow N}{\frac{G \mid \alpha, \square \alpha, M \Rightarrow N, \neg \beta}{G \mid \square \alpha, M \Rightarrow N, \neg \beta} \neg A_{1}} \neg K
\end{aligned}
$$


Let us now consider the permutation with the two premises-rule $(\wedge K)$. We have the following derivation:

$$
\begin{gathered}
\frac{G \mid \alpha, \square \alpha, M \Rightarrow N, \beta}{G \mid \square \alpha, M \Rightarrow N, \beta} \square A_{1} \quad G \mid \square \alpha, M \Rightarrow N, \gamma \\
\frac{\downarrow}{G \mid \square \alpha, M \Rightarrow N, \beta \wedge \gamma} \wedge K \\
\downarrow \\
\frac{G \mid \alpha, \square \alpha, M \Rightarrow N, \beta \quad \frac{G \mid \square \alpha, M \Rightarrow N, \gamma}{G \mid \alpha, \square \alpha, M \Rightarrow N, \gamma} I W}{\frac{G \mid \alpha, \square \alpha, M \Rightarrow N, \beta \wedge \gamma}{G \mid \square \alpha, M \Rightarrow N, \beta \wedge \gamma} \square A_{1}} \wedge K
\end{gathered}
$$

Let us remark that the transformation of the first derivation into the second one is done by means of an application of the height-preserving admissible rule of internal weakening $(I W)$.

Finally we show the permutation in case of the rule $(\square K)$ :

$$
\begin{gathered}
\frac{G|\alpha, \square \alpha, M \Rightarrow N| \Rightarrow \beta}{G|\square \alpha, M \Rightarrow N| \Rightarrow \beta} \square A_{1} \\
\frac{\downarrow}{G \mid \square \alpha, M \Rightarrow N, \square \beta} \\
\frac{G|\alpha, \square \alpha, M \Rightarrow N| \Rightarrow \beta}{G \mid \alpha, \square \alpha, M \Rightarrow N, \square \beta} \square A_{1}
\end{gathered}
$$

凶

Lemma 6.2. The rule $\left(\square A_{2}\right)$ permutes down with respect to the rules $(\neg A)$, $(\neg K),(\wedge A),(\wedge K),\left(\square A_{1}\right)$. It also permutes with the instances of $(\square K)$ when the principal formula $\alpha$ of its premise is not active in the sequent where the principal formula of the premise of $\square K$ occurs.

Proof. The cases where one permutes with the one-premise propositional rules or with the two-premises rule $(\wedge K)$ can be dealt with analogously to the corresponding ones of the previous lemma. We show the permutation in case of the rule $(\square K)$. We underline that this case is constrained by the hypothesis that the principal formula $\alpha$ of the premise of the rule $\left(\square A_{2}\right)$ is not active in the same 
sequent where the principal formula of the premise of the rule ( $\square K)$ occurs. By taking in account this restriction, the permutation is straightforward:

$$
\begin{gathered}
\frac{G|\square \alpha, M \Rightarrow N| \alpha, P \Rightarrow Q \mid \Rightarrow \beta}{G|\square \alpha, M \Rightarrow N| P \Rightarrow Q \mid \Rightarrow \beta} \square A_{2} \\
\frac{\downarrow}{G|\square \alpha, M \Rightarrow N, \square \beta| P \Rightarrow Q} \square K \\
\frac{G|\square \alpha, M \Rightarrow N| \alpha, P \Rightarrow Q \mid \Rightarrow \beta}{G|\square \alpha, M \Rightarrow N, \square \beta| \alpha, P \Rightarrow Q} \square K \\
G|\square \alpha, M \Rightarrow N, \square \beta| P \Rightarrow Q \\
\end{gathered}
$$

$\nabla$

Corollary 6.3. In a minimal derivation in $C S S 5_{s}$, the rule $\left(\square A_{1}\right)$ cannot be applied more than once on the same pair of principal formulas of any branch.

Proof. Let us suppose to have a minimal derivation where the rule $\left(\square A_{1}\right)$ has been applied twice on the same pair of sequents:

$$
\begin{gathered}
\frac{G^{\prime} \mid \alpha, \square \alpha, M^{\prime} \Rightarrow N^{\prime}}{G^{\prime} \mid \square \alpha, M^{\prime} \Rightarrow N^{\prime}} \square A_{1} \\
\cdot \\
\cdot \\
\frac{G \mid \alpha, \square \alpha, M \Rightarrow N}{G \mid \square \alpha, M \Rightarrow N} \square A_{1}
\end{gathered}
$$

By permuting down $\left(\square A_{1}\right)$ with respect to the the steps in the dotted part of the derivation, we obtain a derivation of the same height ending with:

$$
\frac{G \mid \alpha, \alpha, \square \alpha, M \Rightarrow N}{\frac{G \mid \alpha, \square \alpha, M \Rightarrow N}{G \mid \square \alpha, M \Rightarrow N} \square A_{1}}
$$

By applying the height-preserving admissible rule $(C A)$ on the two occurrences of the formula $\alpha$ in place of the upper $\left(\square A_{1}\right)$, we obtain a shorter derivation, contrary to the assumption of minimality. $\otimes$ 
Corollary 6.4. In a minimal derivation in $C S S 5_{s}$, the rule $\left(\square A_{2}\right)$ cannot be applied more than once on the same pair of sequents of any branch.

Proof. Consider a minimal derivation where the rule $\left(\square A_{2}\right)$ has been applied twice on the same pair of sequents:

$$
\begin{gathered}
\frac{G^{\prime}\left|\square \alpha, M^{\prime} \Rightarrow N^{\prime}\right| \alpha, P^{\prime} \Rightarrow Q^{\prime}}{G^{\prime}\left|\square \alpha, M^{\prime} \Rightarrow N^{\prime}\right| P^{\prime} \Rightarrow Q^{\prime}} \square A_{2} \\
\cdot \\
\cdot \\
\cdot \\
\frac{G|\square \alpha, M \Rightarrow N| \alpha, P \Rightarrow Q}{G|\square \alpha, M \Rightarrow N| P \Rightarrow Q} \square A_{2}
\end{gathered}
$$

By permuting down $\left(\square A_{2}\right)$ with respect to the steps in the dotted part of the derivation, we obtain a derivation of the same height ending with:

$$
\frac{G|\square \alpha, M \Rightarrow N| \alpha, \alpha, P \Rightarrow Q}{\frac{G|\square \alpha, M \Rightarrow N| \alpha, P \Rightarrow Q}{G|\square \alpha, M \Rightarrow N| P \Rightarrow Q} \square A_{2}}
$$

By applying the height-preserving admissible rule $(C A)$ on the two occurrences of the formula $\alpha$ in place of the upper $\left(\square A_{2}\right)$, we obtain a shorter derivation, contrary to the assumption of minimality.

Finally we underline that if the principal formulas of the premise of the upper application of the rule $\left(\square A_{2}\right)$ were active in the sequent where the principal formula of the premise of $\square K$ occurs, then that sequent would disappear and therefore we would not find it in the premise of the lower application of the rule $\left(\square A_{2}\right)$. The conclusion is that the restriction of lemma 6.2 is not limitative for the proof of this corollary.

Now we prove that the modal logic $S 5$ is decidable by showing effective bounds on proof search in the calculus $C S S 5_{s}$.

Theorem 6.5. The calculus $C S S 5_{s}$ allows terminating proof search.

Proof. Place an hypersequent $G$, for which we are looking for a proof search, at the root of the procedure. Apply first the propositional rules and then the modal rules. The propositional rules reduce the complexity of the hypersequent. The rule $(\square K)$ removes the modal constant $\square$ and adds a new sequent, each of the rules $\left(\square A_{i}\right)$ increases the complexity. However, by the corollary 6.3 , the rule $\left(\square A_{1}\right)$ cannot be applied more than once on the same pair of principal formulas, 
while, by the corollary 6.4 , the rule $\left(\square A_{2}\right)$ cannot be applied more than once on the same pair of sequents. Therefore the number of applications of the two rules $\left(\square A_{1}\right)$ and $\left(\square A_{2}\right)$ is bounded, respectively, by the number of $\square$ 's occurring in the negative part (see definition below) of the hypersequent to prove, and by the number of the sequents which may appear in the derivation. This derivation, in its turn, is bounded by the number of sequents belonging to the hypersequent to prove, and the sequents which can be introduced by applications of the rule $(\square K)$.

We finally explain how to calculate explicit bounds. First of all define the negative and positive parts of the hypersequent $M_{1} \Rightarrow N_{1}|\ldots| M_{n} \Rightarrow N_{n}$, as the negative and positive parts of each of the following conjuncts and disjuncts:

$$
\bigwedge M_{1} \rightarrow \bigvee N_{1}, \ldots, \bigwedge M_{n} \rightarrow \bigvee N_{n}
$$

For any given hypersequent $G$, let $n(\square)$ be the number of $\square$ 's in the negative part of the hypersequent $G$, and $p(\square)$ be the number of $\square$ 's in the positive part of the hypersequent $G$.

The number of applications of the rule $\left(\square A_{1}\right)$ in a minimal derivation is bounded by:

$$
n(\square)
$$

In the case where the root-hypersequent is just a sequent, the number of applications of the rule $\left(\square A_{2}\right)$ in a minimal derivation is bounded by:

$$
n(\square) \cdot p(\square)
$$

In the case where the root-hypersequent is a hypersequent and $s$ is the number of sequents which occurs in it, the number of applications of the rule $\left(\square A_{2}\right)$ in a minimal derivation is bounded by:

$$
n(\square) \cdot(p(\square)+s)
$$

凶

Acknowledgements I wish to thank Pierluigi Minari for his helpful comments and useful suggestions; Brian Hill for correcting my English.

Francesca Poggiolesi,

Department of Philosophy,

University of Florence,

IHPST,

University of Paris 1, 
Via Bolognese 52,

50139, Florence, Italy.

email: francesca.poggiolesi@unifi.it

\section{References}

Avron, A. (1996). The method of hypersequents in the proof theory of propositional non-classical logic. In W. Hodges, M. Hyland, C. S. e. J. S., editor, Logic: from foundations to applications, pages 1-32. Oxford University Press.

Blumey, S. and Humberstone, L. (1991). A perspective on modal sequent logic. Publications of the Research Institute for Mathematical Sciences, Kyoto University, 27:763-782.

Braüner, T. (2000). A cut-free gentzen formulation of the modal logic s 5. Logic Journal of the IGPL, 8:629-643.

Brünnler, K. (2006). Deep sequent systems for modal logic. Advances in Modal Logic AiML, 6:107-119.

Cerrato, C. (1993). Cut-free modal sequents for normal modal logics. NotreDame Journal of Formal Logic, 34:564-582.

Došen, K. (1985). Sequent systems for modal logic. Journal of Symbolic Logic, 50:149-159.

Indrezejczak, A. (1997). Generalised sequent calculus for propositional modal logics. Logica Trianguli, 1:15-31.

Matsumoto, K. and Ohnishi, M. (1959). Gentzen method in modal calculi. Osaka Mathematical Journal, 11:115-120.

Mints, G. (1997). Indexed systems of sequents and cut-elimination. Journal of Philosophical Logic, 26:671-696.

Negri, S. (2005). Proof analysis in modal logic. Journal of Philosophical Logic, 34:507-534.

Poggiolesi, F. (2006). Sequent calculus for modal logic. Logic Colloqium.

Poggiolesi, F. (2007). Two cut-free sequent calculi for modal logic s5. Submitted to Proceedings of SILFS Conference.

Poggiolesi, F. (2008). The method of tree-hypersequent for modal propositional logic. To appear in Trends in Logic IV, Studia Logica Library.

Restall, G. (2006). Sequents and circuits for modal logic. To appear in the Proceedings of Logic Colloquium. 
Sato, M. (1980). A cut-free gentzen-type system for the modal logic s5. Journal of Symbolic Logic, 45:67-84.

Wansing, H. (1994). Sequent calculi for normal modal propositional logics. Journal of Logic and Computation, 4:125-142. 